\title{
Management for locally advanced cervical cancer: new trends and controversial issues
}

\author{
Oyeon Cho, MD, PhD, Mison Chun, MD, PhD \\ Department of Radiation Oncology, Ajou University School of Medicine, Suwon, Korea
}

This article reviewed new trends and controversial issues, including the intensification of chemotherapy and recent brachytherapy (BT) advances, and also reviewed recent consensuses from different societies on the management of locally advanced cervical cancer (LACC). Intensive chemotherapy during and after radiation therapy (RT) was not recommended as a standard treatment due to severe toxicities reported by several studies. The use of positron emission tomography-computed tomography (PET-CT) and magnetic resonance imaging (MRI) for pelvic RT planning has increased the clinical utilization of intensity-modulated radiation therapy (IMRT) for the evaluation of pelvic lymph node metastasis and pelvic bone marrow. Recent RT techniques for LACC patients mainly aim to minimize toxicities by sparing the normal bladder and rectum tissues and shortening the overall treatment time by administering a simultaneous integrated boost for metastatic pelvic lymph node in pelvic IMRT followed by MRI-based image guided adaptive BT.

Keywords: Uterine cervical neoplasms, Intensity-modulated radiotherapy, Drug therapy, Brachytherapy

\section{Introduction}

The main treatment for early-stage cervical cancer is radical surgery or primary radiation depending on the patients' general condition. Radiation therapy (RT) has been the main treatment option for locally advanced cervical cancer (LACC) since the discovery of radiation. LACC is defined as a tumor categorized as stage IB2 and higher since the rate of lymph node metastasis is quite high. In 1999, the National Cancer Institute mailed a clinical announcement to thousands of physicians stating that strong consideration would be given to adding chemotherapy (CTX) to radiation in the treatment of invasive cervical cancer based on the results of five randomized clinical trials (RCTs) [1-5].

The present article reviewed the new trends and controversial issues such as the intensification of CTx and recent advances in brachytherapy (BT). It also reviewed the recent consensus on the management of LACC from different societies.

\section{Prognostic Factors Affecting Survival and Local Control in LACC}

Conventionally, staging and nodal involvement are prognostic factors for cure in cervical cancer. Bae et al. [6] reviewed 397 LACC patients treated with definitive platinum-based concurrent chemoradiotherapy (CCRT). Their risk factors for local control (LC) and survival were large tumors (>5 cm), young age ( $\leq 40$ years), non-squamous histology, positive node on magnetic resonance imaging (MRI), and advanced stage

Received 07 November 2018, Revised 13 December 2018, Accepted 17 December 2018.

Correspondence: Mison Chun, MD, PhD, Department of Radiation Oncology, Ajou University School of Medicine, 164, Worldcup-ro, Yeongtong-gu, Suwon 16499, Korea. Tel: +82-32-219-5884, Fax: +82-31-219-5894, E-mail: chunm@ajou.ac.kr (http://orcid.org/0000-0003-2334-3316)

(c) This is an Open Access article distributed under the terms of the Creative Commons Attribution Non-Commercial License (http://creativecommons.org/ licenses/by-nc/4.0/) which permits unrestricted non-commercial use, distribution, and reproduction in any medium, provided the original work is properly cited.

www.e-roj.org 
(III-IV). They defined the high-risk group as follows: (1) tumor size larger than $5 \mathrm{~cm}$ and at least one other risk factor or (2) tumor size $5 \mathrm{~cm}$ or less and at least three other risk factors. The 3-year locoregional failure (LRF) rates for the high- and low-risk groups were $26 \%$ and $7 \%$, respectively. The 3 -year overall survival (OS) rate also differed significantly between these groups, at 57\% and $86 \%$, respectively. Cervical cancer in young age groups is considered an aggressive form with increased positive nodal metastasis [7]. Another recently reported negative prognostic factor is increased standardized uptake value (SUV) in pre-therapeutic positron emission tomography-computed tomography (PET-CT) [8].

Human papillomavirus (HPV) infection in cervical cancer patients also affects $L C$ and survival. A systematic review reported that HPV genotypes result in differential prognoses in cervical cancer patients even after adjusting for staging, tumor size, and tumor grade [9-12]. Pre-treatment systemic markers, such as anemia, thrombocytosis, leukocytosis, and lymphopenia, are negative prognosticators in cervical cancer patients treated with CCRT [13-16]. Anemia and lymphopenia during CCRT and lymphopenia 2 months after initiating CCRT were associated with increased risk of progression and poor survival $[15,17,18]$.

Klopp and Eifel [19] reviewed the biological predictors of response to $R T$ in patients with cervical cancer. A variety of biomarkers were identified, such as clinical (staging including tumor size, presence of nodal metastasis) and morphologic predictors (tumor histology), non-molecular biomarkers (hypoxia, interstitial tumor pressure, vascular density, hemoglobin level, and fludeoxyglucose-PET avidity), and molecular biomarkers (single-gene or multigene biomarkers, type of HPV positivity). They concluded that the results were mixed regarding the predictive value of single-gene biomarkers and that multigene predictors remain immature. However, they stated that the most useful biomarkers in the future will identify patients who will benefit from additional or less RT or CTx.

Although various variables have been investigated to predict the treatment outcomes of LACC patients, the current results are not conclusive enough to reach an individual risk stratification beyond the existing staging system.

\section{CCRT is Better Than RT Alone}

Updated results from the Radiation Therapy Oncology Group (RTOG) 90-01 trial, with a median follow-up of 6.6 years in patients with high-risk cervical cancer, revealed that the OS rate of patients treated with CCRT was significantly higher than that of patients treated with extended field radiotherapy (EFRT) (67\% vs. $41 \%$ at 8 years; $p<0.0001)$. There was an overall reduction of $51 \%$ in the risk of disease recurrence (95\% confidence interval $[\mathrm{Cl}], 36 \%-66 \%)$ in patients who received CCRT [20]. However, these studies included early-stage patients and hydroxyurea with radiation was administered in the control arm. A review in 2008 including studies comparing CCRT to radiation (with or without surgery and some data on adjuvant CTx) reported a trend in the relative effect of CCRT by stage, with a decreasing benefit of CCRT with increasing stage [21]. The benefit was only 7\% in stage IIB and 3\% in stages III-IVA disease. The review also found an increased benefit in patients treated with additional CTx after CCRT from two small studies. To address the limitations of the variety of stages and different CTx schedules, Datta et al. [22] performed a metaanalysis exclusively of patients with LACC who received either CCRT or RT only without surgical intervention. The results confirmed that CCRT significantly improves outcomes in LACC, with complete remission and locoregional control (LRC) rates of $10.2 \%$ and $8.4 \%$, respectively, and an OS of $7.5 \%$, but also with a 10.4\% higher incidence of grade III/IV acute toxicities. The occurrences of late toxicities in both groups were equivalent. There was no difference in the outcomes between weekly or 3-weekly cisplatin (CDDP) regimens.

Although CCRT is the standard treatment for LACC, there is a possibility to improve the survival rate, especially in patients with stage IIB-IVA disease.

\section{Delivery of Chemotherapy to Enhance Treatment Results in LACC}

Since the early 1990s, CDDP during RT has been the standard treatment for LACC. Schmid et al. [23] found that the number of CTx cycles did not significantly impact the distant metastasis-free survival in the low-risk group ( $p=0.782$ ). However, in the high-risk group (stage III or IVA, any stage and positive nodes) ( $p=0.022)$, there were increased distant metastases (DMs) with a decreasing number of CTx cycles (fewer than five cycles). Early analysis from image-guided intensity-modulated external beam radio-CTx and MRI-based adaptive BT in a locally advanced cervical cancer (EMBRACE) I trial also showed higher DMs in node-positive and advancedstage patients who received fewer than five cycles of CTx [24].

Based on their analysis of four randomized trials and four retrospective studies (including two studies with postoperative CTx), Petrelli et al. [25] concluded that platinum-based 
combination therapy with radiation is a better option for improved progression-free survival (PFS) and OS rates, by close to $30 \%$, than weekly CDDP alone with RT in LACC. Their results were the same even after excluding surgical series. However, toxicities were generally underreported, and the incidence of neutropenia, gastrointestinal (GI), and genitourinary (GU) toxicities is much higher in platinum-based doublet CTX than in single CTx with RT. A randomized trial by the Asian Gynecologic Oncology Group evaluated outcomes between CCRT with CDDP alone and with both CDDP and gemcitabine in LACC [26]. Interim analysis showed no difference in the PFS rates $(65.1 \%$ vs. $71 \%, p=0.71)$ and $0 S$ rates at three years (74.1\% vs. 85.9\%, $p=0.89$ ), with increased toxicities. They closed the study early after accruing only 74 patients.

Choi et al. [27] performed a matched-case comparison between CCRT and CCRT followed by consolidation CTX (5$\mathrm{FU}$ and CDDP three cycles). The rate of distant recurrence was lower in the group that received consolidation CTx without any difference in LRC and the OS rate was higher $(70.1 \%$ vs. $55.1 \%, p=0.079$ ). Tang et al. [28] reported the outcome of a RCT comparing usual CCRT versus one cycle of neoadjuvant paclitaxel and CDDP and CCRT followed by two cycles of CTx for the treatment of adenocarcinoma (stage II-IVA). The group that received CTx with CCRT showed excellent 5-year relapse-free survival (71.4\%) and OS (74.3\%) rates. A report on adjuvant CTX after CCRT (CDDP and ifosfamide during and after CCRT) showed low distant failure (13.4\%) and local failure (2.5\%) rates [29]. However, a four-arm randomized trial by Lorvidhaya et al. [30] did not confirm the benefit of adjuvant CTx (arm 1, RT alone; arm 2, RT with adjuvant CTx; arm 3, CCRT; arm 4, CCRT with adjuvant CTX). The trial used mitomycin $\mathrm{C}$ and oral 5-FU during radiation and oral 5-FU for adjuvant CTx. A Cochrane review by Tangjitgamol et al. [31] did not reach a conclusion due to inconsistent data regarding the benefit of adjuvant $\mathrm{CT} x$.

A randomized study by Duenas-Gonzales et al. [32] compared CCRT to the combination of CDDP and gemcitabine followed by two cycles of adjuvant CDDP and gemcitabine with conventional CCRT. They showed that the PFS at 3 years was significantly improved in the gemcitabine arm versus that in the standard arm (74.4\% vs. $65.0 \%, p=0.029)$. There were increased occurrences of grade 3 and 4 toxicities, which were manageable. In subgroup analysis, there was an increased benefit in patients with higher-stage disease (stages III-IVA vs. stage IIB), which suggested that the increased toxic effects of the experimental protocol may be justified for these patients [33]. The OUTBACK trial (ANZGOG 0902/G0G 0274/RTOG
1174) is an ongoing RCT of adjuvant CTx following CCRT. The standard arm is RT with five cycles of weekly CDDP, while the experimental arm includes the same CCRT followed by four cycles of 3-weekly carboplatin and paclitaxel.

Although neoadjuvant chemotherapy (NAC) followed by radical hysterectomy for $L A C C$ has been considered as an alternative of CCRT in stage IB-IIA, recently published RCT result proved that NAC group had inferior 5-year disease-free survival (DFS) compared with CCRT group (69.3\% vs. 76.7\%, $p=0.038$ ) [34]. However, the role of NAC in CCRT has not been identified in LACC. Recent phase II trial did not show any meaningful improvement in treatment outcome [35]. The INTERLACE study (UK-funded) is an ongoing phase III trial of weekly induction CTx (carboplatin and paclitaxel) followed by standard CCRT compared to standard CCRT alone.

The benefit of novel target agents such as antiangiogenic blockage (bevacizumab) and immunotherapy (pembrolizumab) for LACC treatment has been tested or is under evaluation in various clinical trials [36]. Phase II study adding bevacizumab to pelvic CCRT for LACC (RTOG 0417) showed good treatment results (3-year OS, DFS, and LRF rate: $81.3 \%, 68.7 \%$, and $23.2 \%$, respectively) [37]. A randomized phase II study of CCRT and pembrolizumab is enrolling patients (ClinicalTrials.gov identifier: NCT02635360).

The risk-benefit of adding (neo)adjuvant and/or concomitant CTx to conventional CCRT for LACC remains inconclusive despite several positive reports. We look forward to the results of the two ongoing RCTs. Although clinical evaluations of targeted agents to enhance RT outcome for LACC are in the beginning phase, targeted agents are promising for achieving low toxicity and high survival combined with RT.

\section{Is Intensity-Modulated Radiation Therapy Essential?}

Several reports on intensity-modulated radiation therapy (IMRT) for cervical cancer showed fewer acute GI and GU toxicities and even fewer late GI toxicities [38-40]. Naik et al. [41] reported the results of an institutional randomized study comparing IMRT and three-dimensional (3D) conformal pelvic RT with concurrent CDDP. A total of 40 patients were randomized to the treatment groups. The IMRT plans were superior to the 3D conformal plans in that they reduced volume receiving high doses in the bladder, rectum, bowel, and bone marrow (BM). Clinically, there was no significant difference in hematological toxicities, while the acute $\mathrm{Gl}$ and GU toxicities reflected the comparative advantage of the IMRT 
plans. In a detailed study using PET-CT, the occurrence of severe acute neutropenia ( $\geq$ grade 3 ) was lower in patients who received IMRT planning with PET-CT than that in patients who received the usual IMRT plan without PET-CT evaluation for functional BM [42].

Due to the significant variability in the incidence of hematologic toxicities even in patients with BM-sparing IMRT, Noticewala et al. [43] conducted a longitudinal study to verify the compensatory response in the outside radiation field BM activity. They showed that patients have different subacute compensatory responses after CCRT; compared to those treated with weekly cisplatin alone, patients treated with cisplatin and gemcitabine had a lower mean SUV in the extrapelvic structures as well as a lower compensatory response. This study emphasized the importance of considering the potential increase in severe toxicities in LACC patients treated with CCRT including more intensive CTx regimen, especially in older patients and those with more morbidities.

Another issue in using IMRT on a routine basis is organ motion during RT (intra-fraction) and during the course of RT (inter-fraction). Jadon et al. [44] reported a systematic review of organ motion in external beam radiation therapy (EBRT) for cervical cancer. Given the steep dose gradients around the planning target volumes in IMRT planning, the degree of movement during radiation treatment should be considered. In total, 448 studies were identified and screened to find 39 relevant studies, 12 of which were abstracts. These studies showed that within the target volume for cervical cancer RT, uterine motion is greater than cervical motion. Uterine and cervical motions are predominantly influenced by bladder and rectal filling, respectively. Organ motion patterns are patient-specific. The potential solutions include anisotropic margins with increased margins in the anteroposterior and superio-inferior directions or greater planning target volume (PTV) margins around the uterine fundus than around the cervix. As pelvic organ motion seems to be patient-specific, individualized PTV margins and adaptive image-guided radiation therapy (IGRT) have also been recommended to ensure target volume coverage while increasing the sparing of the organs-at-risk (OAR). Experts in gynecological radiation oncology provided guidelines for the clinical target volume (CTV) definition of IMRT for the definitive treatment of cervical cancer [45].

As IMRT planning can spare normal organs such as the bladder, intestines, and BM better than conventional 3D conformal plan and is vulnerable to organ motion, IMRT plans and simultaneous integrated boost (SIB) of LN metastasis instead of SIB of primary cervical lesion during pelvic CCRT is reasonable. This could be an optimal strategy to reduce RT-induced complications and improve treatment outcome through a shorter overall treatment time (OT).

\section{BT is Mandatory unless There Are Technical Problems}

The standard treatment for LACC has been established for a long period of time as CCRT with BT boost. Recent advances in $B T$, such as image-guided adaptive brachytherapy (IGABT), have increased the local control rate (LCR) to between 79\% and 96\% [46-51]. Charra-Brunaud et al. [50] reported that 3D-based BT had a higher 2-year LCR and lower grade 3-4 toxicity rate than 2D-based BT in CCRT for LACC (LCR, 73.6\% vs. $78.5 \%$; toxicities, $22.7 \%$ vs. $2.6 \%$ ). Two studies using MRIbased BT reported excellent 3-year LCR of 77\%-86\% even in stage IIIb patients $[46,47]$. Advances in radiation techniques have resulted in two trends over the last decade, including the decreasing use of BT and the incremental utilization of $3 D$ BT. In the SEER database, the BT utilization rate has decreased from 83\% in 1988 to $58 \%$ in $2009(p<0.001)$ and the OS was lower in patients without BT (58\% vs. $46 \%$ at 4 years) [52]. Gill et al. [53] also found a significantly reduced use of BT with an increasing use of IMRT or SBRT in the National Cancer Database between 2004 and 2011 despite BT being a critical component of treatment of LACC.

In the United States, there was an increased use of imagebased BT between 2007 and 2014, particularly the use of MRI and volume-based dose delineation to the target (14\%-52\%) [54]. This advance in IGABT-allowed doses could be prescribed to volumes with better delineation and coverage of target volume and with a lower dose to the OAR. In other words, this technique is response-adaptive RT. Potter et al. [55] from the Groupe Européen de Curiethérapie-European Society for Radiotherapy \& Oncology (GEC-ESTRO) described the details of reporting the residual gross tumor, adaptive high-risk target, intermediate-risk target, and adaptive low-risk target volumes on T2-weighted MRI at the time of starting BT. For the whole treatment, they recommended that the total dose values be reported as the physical dose, indicating the fractionation and dose rate, as well as the biologically weighted dose (equivalent dose to 2 Gy per fraction [EQD2]). In 2007, Potter et al. [56] reported that MRI-assisted adaptive BT was more effective in LC in tumors larger than $5 \mathrm{~cm}$. The American Brachytherapy Task Group [57] reported the pooled analysis of outcomes for high-dose BT for cervical cancer. In their analysis, 57 studies 
used point A prescription, while 10 studies used image-based BT. Comparison of patients receiving CCRT and IGABT to those receiving traditional point $A$ prescription revealed significant improvement in pelvic control and DFS in IGABT.

The EMBRACE study, a prospective observational trial, introduced MRI-based BT in LACC patients in multiple centers worldwide treated according to the GEC-ESTRO guidelines. The RetroEMBRACE study report of 731 patients with LACC from 12 centers showed that IGABT in combination with CCRT led to an excellent LCR of 91\%, a pelvic control rate of $87 \%$, and an OS of 74\% with limited severe toxicities (less than 7\%). Even in stage IIIB, the LCR at 5 years was 75\% [58]. In addition, the D90 to the high-risk clinical target volume $(C T V H R)(p=0.022)$ was significant for LC, whereas increasing CTVHR volume and longer OTT were associated with a worse LC. Histology $(p=0.084), C T x(p=0.49)$, and dose rate $(p=1.00)$ did not significantly impact the LC [59]. A dose of 5 Gy (CTVHR) is required to compensate for a 1-week increase in $0 \pi$. Increased CTVHR volume by $10 \mathrm{~cm} 3$ requires an additional $5 \mathrm{~Gy}$ for an equivalent LC. They recommended that EBRT and BT should be finished within 7 weeks.

The EMBRACE II study report was released in 2018 and reviewed the outcome and prospect of two decades of evolution within the GEC-ESTRO GYN working group and EMBRACE studies [24]. The EMBRACE II was initiated in April 2016 (https://www.embracestudy.dk) and defines interventions that address local, nodal, and systemic treatment as well as OAR exposure. The EMBRACE II prescribed MRIguided adaptive BT with combined intracavitary/interstitial techniques and specific dose volume constraints for adaptive targets and OARs and image-guided EBRT for specific targets and techniques (IMRT, IGRT, and SIB for nodal disease) and concomitant radio-CTX. IGABT is a mainstream component of LACC treatment due to its precise delivery of dose to the target volume and excellent sparing of OAR. In addition, this advantage of IGABT can contribute to the reduction of OTT by increasing the fraction size. The only difficulty in using IGABT is that it is time-consuming. Kim et al. [60] reported that mean total procedure time is $149.3 \pm 17.9$ minutes (range, 112 to 178 minutes). It requires a multidisciplinary team including an anesthesiologist, a radiation oncologist, nurses, and a radiation therapist.

IGABT that can deliver high radiation dose to large target more than $4-5 \mathrm{~cm}$ is a remarkable technical improvement influencing treatment outcome and toxicities through reduction in OTT and OAR sparing.

https://doi.org/10.3857/roj.2018.00500

\section{Non-brachytherapy Boost for LACC}

In certain cases, stereotactic ablative body radiotherapy (SABR) may be an option. Mendez et al. [61] reviewed 375 gynecological cancer patients treated with SABR in 33 reports. SABR was delivered using a CyberKnife, linear accelerator, or tomotherapy. Among them, six studies included cervical cancer patients (33 patients) in place of standard treatment with BT [62-67]. The combined LC was 91\% with a median follow-up time of 6-22 months. However, most studies only included small numbers of patients with short follow-up times. Mazzola et al. [68] applied an integrated boost using volumetric-modulated arc therapy in 30 LACC patients over 70 years of age. They delivered 66 Gy to the macroscopic disease and $54 \mathrm{~Gy}$ to the pelvic nodes in 30 fractions with a median OT of 42 days. The 3 -year OS and LC rates were 93\% and $80 \%$, respectively ( $91 \%$ in stage $\mathrm{II}, 67 \%$ in stage III), with a median follow-up of 32 months. Only two patients had grade 2 rectal toxicities. Non-BT boost is complicated by internal organ motion during treatment. Thus, BT boost should be used routinely unless patients have a difficult anatomy or medical contraindication to sedation or they refuse BT.

In Korea, number of BT centers compared with radiation oncology centers have decreased since 2006 due to low medical reimbursement, expensive source, and shortage of human resources [69]. Seven of 42 centers that did not practice BT treated some patients with EBRT alone. Thus, shortage of resources can make physicians use EBRT instead of BT.

BT is an essential part for the treatment of LACC. However, there are alternative options including SABR or SIB in exceptional cases.

\section{Review of Updated Cervical Cancer Management Guidelines}

The American Brachytherapy Society (ABS) consensus guideline for LACC reviewed the important points in the management of cervical cancer to improve treatment results [70]. Advanced imaging study is important. MRI shows excellent views of tumor extension, while PET-CT adds information on nodal metastasis. The finding of pelvic and/or para-aortic lymph node (PALN) involvement may be used to guide clinical decisions related to radiation treatment planning, such as the field size and delivered dose. Lymph node staging by PETCT has also been shown to be an important prognostic factor for recurrence and survival. Surgical staging before definitive 
chemoradiotherapy is also an acceptable approach to detect intra-abdominal disease and for pathologic assessment or debulking of pelvic and/or PALNs. They recommended EBRT to deliver 45 Gy to the initial target volume with an additional boost to the involved nodes (60-70 Gy in a combination of EBRT and BT). In addition, the OTT should be limited to 8 weeks. BT is not an option and it should be done as much as they can.

Recently, the ESGO/ESTRO/ESP published online guidelines on cervical cancer [71]. This article reviews radiation for LACC.

In the staging process, pelvic MRI is mandatory in the initial workup for the assessment of pelvic tumor extent. Cystoscopy or sigmoidoscopy may be considered for biopsy of suspicious lesions in the urinary bladder or rectum detected by MRI. There have also been advances in the evaluation of nodal spread. In LACC or early-stage disease with suspicious lymph nodes on imaging, PET-CT or chest/abdomen CT are recommended. Due to the high false-negativity of PALN involvement in LACC, even PALN dissection, at least up to the inferior mesenteric artery, may be considered in the cases negative in PET-CT.

A randomized trial from French comprehensive cancer centers showed the excellent outcome of 3-year event-free survival (EFS) rate of 69\% in patients with negative PET scans and para-aortic microscopic metastasis $\leq 5 \mathrm{~mm}$ detected by surgical staging and treated with para-aortic CCRT with concomitant CDDP, while patients with negative PET and paraaortic metastasis more than $5 \mathrm{~mm}$ had a 3-year EFS rate of $17 \%$ [72]. The false-negative rate was $12 \%$ in PET-CT for PALN involvement and an even higher rate of $22 \%$ in patients with involved pelvic node without uptake on para-aortic at PETCT. This meant that that surgical para-aortic staging was associated with survival. A recent multicenter cohort analysis from France, reporting that nodal surgical staging was an independent prognostic factor for survival, supported the need of para-aortic surgical staging [73].

The ESGO/ESTRO recommended definitive platinum-based CCRT and BT to avoid the combination of radical surgery and postoperative external RT because of the significant increase in morbidity and no evidence of improved survival. They emphasized keeping the 0T to within 7-8 weeks, especially in patients with positive nodes who require nodal boosts. Boost treatment may be applied as SIB at the time of pelvic RT. For $E B R T$ and $B T, I G R T$ is strongly recommended to reduce the dose to the normal tissue and to ensure the full dose coverage to the residual tumor at the completion of EBRT.

The ESTRO recommendations included the following points: a total dose to the involved nodes, including the contribution from $\mathrm{BT}$, of 55 to $60 \mathrm{~Gy}$ (EQD2). IGABT is recommended preferably with the use of MRI prior to the initiation of $B T$. In IGABT, the dose to the intermediate-risk clinical target volume should ideally be a total 85-90 Gy (EOD2). To reduce the 0T, BT should be started as soon as possible. ESTRO suggested that BT can be delivered within 1-2 weeks toward the end of or after CCRT in cases of large tumors. In smaller tumors, BT may start earlier during CCRT. They strongly discouraged the use of a midline block to boost parametrium and recommended the use of an external beam to administer an extra dose to the residual tumor in advanced IGRT, especially beyond 45-50 Gy.

Pelvic IMRT using IGRT with nodal SIB to reduce OTT and toxicities were recommended. Exact dose delivery to the residual tumor and risk region through IGABT was recommended and a fast start of BT was recommended for fast completion of treatment.

Pelvic MRI and PET-CT are a common practice for evaluation of disease extent and LN metastasis. Para-aortic surgical staging even in negative PET-CT is suggested. IMRT using IGRT followed by IGABT is recommended for reduction of OTT and toxicities.

\section{Aging and Management of Cervical Cancer}

Aging patients (older populations) were defined as those aged 65 years or older. Moore et al. [74] analyzed cervical cancer patients treated with primary CCRT on GOG protocols (GOG 113, GOG 120, GOG 165, and GOG 219) stratified by decade of age. The age groups were compared for CCRT tolerance and completion. There were no statistically significant differences in the number of cycles of CTx received, CTx dose modification, CTx dose delay, or completion of the assigned CTx (no difference by age in CTx completion or CTx delays). There was a trend in the oldest age group ( $>70$ years) to receive a lower mean dose to point $A$. There were more major radiation protocol deviations among the oldest age group due to a decreased completion of BT (30\% in those $\geq 70$ years compared to $13 \%$ in those $<40$ years). Sharma et al. [75] reported declining BT use with age in the SEER database, from $66.7 \%$ in those $<50$ years to $58.9 \%$ in those $70-79$ years and $46.3 \%$ in women $>70$ years of age. There were no significant differences in toxicities by age, except for any grade of lymphatic disorders, which were more common with increasing age [74]. Therefore, old age is not a contraindication of standard treatment for LACC. 


\section{Conclusion}

The few changes from conventional to currently recommended CCRT are summarized in Table 1.

In the 21st century, MRI/PET-CT is usually used to aid in staging and LN evaluation of cervical cancer. MRI is valuable for the evaluation of the degree of the local extension cervical tumors as well as for introducing IGABT use in treatment planning for BT. The use of PET-CT and MRI for pelvic RT planning increased the clinical utilization of IMRT for the evaluation of pelvic LN metastasis and pelvic BM. The current orientation of RT for LACC patients is to reduce both toxicities and OTT by using pelvic IMRT and MRI-based IGABT. This is intended to improve treatment results by delivering the planned radiation dose in a short OT. However, it is not easy to apply these strategies to all LACC patients because treatment using MRI-based IGABT at every fraction is money- and timeconsuming.

In limited cases for which BT is not possible due to medical or facility issues, non-BT SABR or SIB can be substituted for BT.

\section{Conflict of Interest}

No potential conflict of interest relevant to this article was reported.

\section{References}

1. Whitney CW, Sause W, Bundy BN, et al. Randomized comparison of fluorouracil plus cisplatin versus hydroxyurea as an adjunct to radiation therapy in stage IIB-IVA carcinoma of the cervix with negative para-aortic lymph nodes: a Gynecologic Oncology Group and Southwest Oncology Group study. J Clin Oncol 1999;17:1339-48.

2. Morris M, Eifel PJ, Lu J, et al. Pelvic radiation with concurrent chemotherapy compared with pelvic and para-aortic radiation for high-risk cervical cancer. N Engl J Med 1999;340:1137-43.

3. Peters WA 3rd, Liu PY, Barrett RJ 2nd, et al. Concurrent chemotherapy and pelvic radiation therapy compared with pelvic radiation therapy alone as adjuvant therapy after radical surgery in high-risk early-stage cancer of the cervix. J Clin Oncol 2000;18:1606-13.

4. Keys HM, Bundy BN, Stehman FB, et al. Cisplatin, radiation, and adjuvant hysterectomy compared with radiation and adjuvant hysterectomy for bulky stage IB cervical carcinoma. N Engl J Med 1999;340:1154-61.

5. Rose PG, Bundy BN, Watkins EB, et al. Concurrent cisplatin-

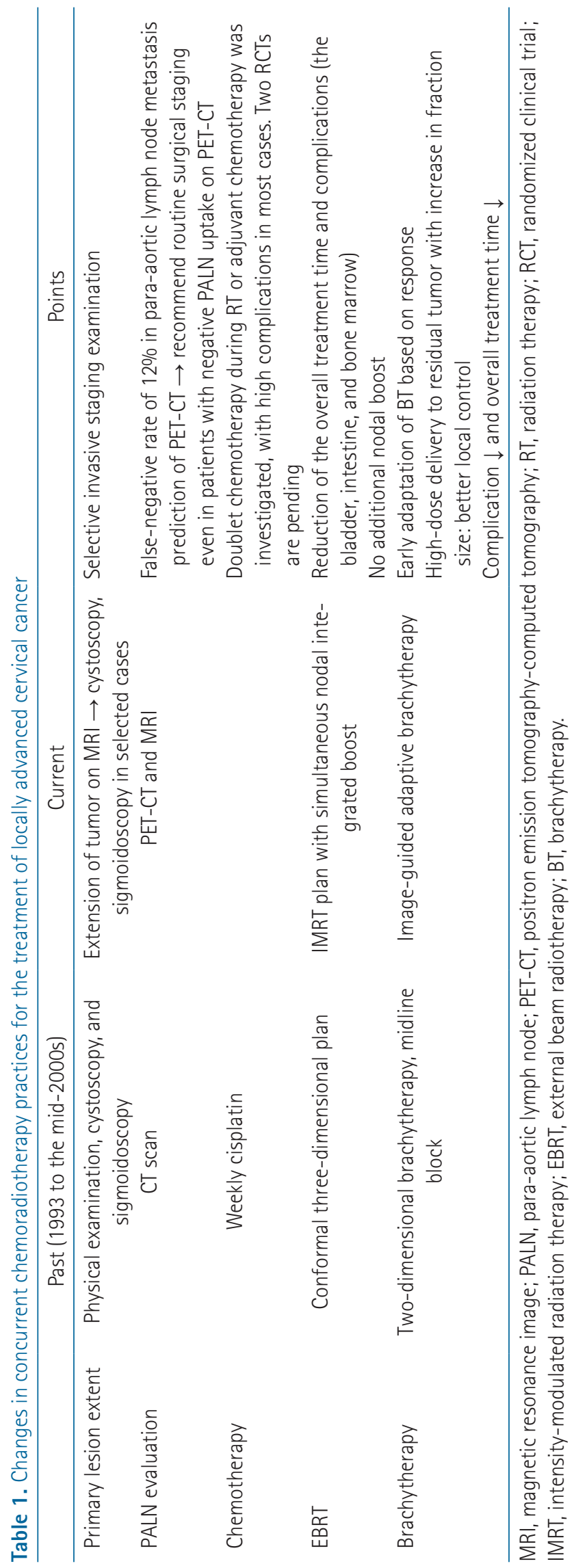

www.e-roj.org

260 
based radiotherapy and chemotherapy for locally advanced cervical cancer. N Engl J Med 1999;340:1144-53.

6. Bae HS, Kim YJ, Lim MC, et al. Predictors of radiation field failure after definitive chemoradiation in patients with locally advanced cervical cancer. Int J Gynecol Cancer 2016;26:73742.

7. Han $\mathrm{CH}, \mathrm{Cho} \mathrm{HJ}$, Lee SJ, et al. The increasing frequency of cervical cancer in Korean women under 35. Cancer Res Treat 2008;40:1-5.

8. Ramlov A, Kroon PS, Jurgenliemk-Schulz IM, et al. Impact of radiation dose and standardized uptake value of (18)FDG PET on nodal control in locally advanced cervical cancer. Acta Oncol 2015;54:1567-73.

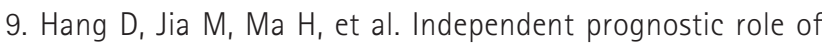
human papillomavirus genotype in cervical cancer. BMC Infect Dis 2017;17:391.

10. Joo J, Shin HJ, Park B, et al. Integration pattern of human papillomavirus is a strong prognostic factor for disease-free survival after radiation therapy in cervical cancer patients. Int J Radiat Oncol Biol Phys 2017;98:654-61.

11. Wang CC, Lai CH, Huang YT, Chao A, Chou HH, Hong JH. HPV genotypes predict survival benefits from concurrent chemotherapy and radiation therapy in advanced squamous cell carcinoma of the cervix. Int J Radiat Oncol Biol Phys 2012;84:e499-506.

12. Li P, Tan Y, Zhu LX, et al. Prognostic value of HPV DNA status in cervical cancer before treatment: a systematic review and meta-analysis. Oncotarget 2017;8:66352-9.

13. Koulis TA, Kornaga EN, Banerjee R, et al. Anemia, leukocytosis and thrombocytosis as prognostic factors in patients with cervical cancer treated with radical chemoradiotherapy: a retrospective cohort study. Clin Transl Radiat Oncol 2017;4:516.

14. Cho Y, Kim KH, Yoon HI, Kim GE, Kim YB. Tumor-related leukocytosis is associated with poor radiation response and clinical outcome in uterine cervical cancer patients. Ann Oncol 2016;27:2067-74.

15. Wu ES, Oduyebo T, Cobb LP, et al. Lymphopenia and its association with survival in patients with locally advanced cervical cancer. Gynecol Oncol 2016;140:76-82.

16. Serkies K, Badzio A, Jassem J. Clinical relevance of hemoglobin level in cervical cancer patients administered definitive radiotherapy. Acta Oncol 2006;45:695-701.

17. Cho O, Chun M, Oh YT, et al. Prognostic implication of simultaneous anemia and lymphopenia during concurrent chemoradiotherapy in cervical squamous cell carcinoma. Tumour Biol 2017;39:1010428317733144.
18. Cho O, Chun M, Chang SJ, Oh YT, Noh OK. Prognostic value of severe lymphopenia during pelvic concurrent chemoradiotherapy in cervical cancer. Anticancer Res 2016:36:3541-7.

19. Klopp AH, Eifel PJ. Biological predictors of cervical cancer response to radiation therapy. Semin Radiat Oncol 2012;22:143-50.

20. Eifel PJ, Winter K, Morris M, et al. Pelvic irradiation with concurrent chemotherapy versus pelvic and para-aortic irradiation for high-risk cervical cancer: an update of radiation therapy oncology group trial (RTOG) 90-01. J Clin Oncol 2004;22:872-80.

21. Chemoradiotherapy for Cervical Cancer Meta-Analysis Collaboration. Reducing uncertainties about the effects of chemoradiotherapy for cervical cancer: a systematic review and meta-analysis of individual patient data from 18 randomized trials. J Clin Oncol 2008;26:5802-12.

22. Datta NR, Stutz E, Liu M, et al. Concurrent chemoradiotherapy vs. radiotherapy alone in locally advanced cervix cancer: a systematic review and meta-analysis. Gynecol Oncol 2017;145:374-85.

23. Schmid MP, Franckena M, Kirchheiner $K$, et al. Distant metastasis in patients with cervical cancer after primary radiotherapy with or without chemotherapy and image guided adaptive brachytherapy. Gynecol Oncol 2014;133:256-62.

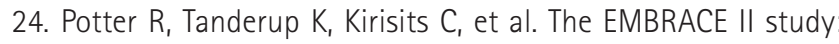
the outcome and prospect of two decades of evolution within the GEC-ESTRO GYN working group and the EMBRACE studies. Clin Transl Radiat Oncol 2018;9:48-60.

25. Petrelli F, De Stefani A, Raspagliesi F, Lorusso D, Barni S. Radiotherapy with concurrent cisplatin-based doublet or weekly cisplatin for cervical cancer: a systematic review and meta-analysis. Gynecol Oncol 2014;134:166-71.

26. Wang CC, Chou HH, Yang LY, et al. A randomized trial comparing concurrent chemoradiotherapy with single-agent cisplatin versus cisplatin plus gemcitabine in patients with advanced cervical cancer: an Asian Gynecologic Oncology Group study. Gynecol Oncol 2015;137:462-7.

27. Choi $\mathrm{CH}$, Lee $\mathrm{YY}, \mathrm{Kim} M \mathrm{M}_{\text {, et }}$ al. A matched-case comparison to explore the role of consolidation chemotherapy after concurrent chemoradiation in cervical cancer. Int J Radiat Oncol Biol Phys 2011;81:1252-7.

28. Tang J, Tang Y, Yang J, Huang S. Chemoradiation and adjuvant chemotherapy in advanced cervical adenocarcinoma. Gynecol Oncol 2012;125:297-302.

29. Jelavic TB, Mise BP, Strikic A, Ban M, Vrdoljak E. Adjuvant chemotherapy in locally advanced cervical cancer after 
treatment with concomitant chemoradiotherapy: room for improvement? Anticancer Res 2015;35:4161-5.

30. Lorvidhaya V, Chitapanarux I, Sangruchi S, et al. Concurrent mitomycin C, 5-fluorouracil, and radiotherapy in the treatment of locally advanced carcinoma of the cervix: a randomized trial. Int J Radiat Oncol Biol Phys 2003;55:122632.

31. Tangjitgamol S, Katanyoo K, Laopaiboon M, Lumbiganon P, Manusirivithaya S, Supawattanabodee B. Adjuvant chemotherapy after concurrent chemoradiation for locally advanced cervical cancer. Cochrane Database Syst Rev 2014;(12):CD010401.

32. Duenas-Gonzalez A, Zarba JJ, Patel F, et al. Phase III, openlabel, randomized study comparing concurrent gemcitabine plus cisplatin and radiation followed by adjuvant gemcitabine and cisplatin versus concurrent cisplatin and radiation in patients with stage IIB to IVA carcinoma of the cervix. J Clin Oncol 2011;29:1678-85.

33. Duenas-Gonzalez A, Orlando M, Zhou Y, Quinlivan $M$, Barraclough $\mathrm{H}$. Efficacy in high burden locally advanced cervical cancer with concurrent gemcitabine and cisplatin chemoradiotherapy plus adjuvant gemcitabine and cisplatin: prognostic and predictive factors and the impact of disease stage on outcomes from a prospective randomized phase III trial. Gynecol Oncol 2012;126:334-40.

34. Gupta S, Maheshwari A, Parab P, et al. Neoadjuvant chemotherapy followed by radical surgery versus concomitant chemotherapy and radiotherapy in patients with stage IB2, IIA, or IIB squamous cervical cancer: a randomized controlled trial. J Clin Oncol 2018;36:1548-55.

35. de Azevedo CRAS, Thuler LCS, de Mello MJG, et al. Phase II trial of neoadjuvant chemotherapy followed by chemoradiation in locally advanced cervical cancer. Gynecol Oncol 2017;146:5605.

36. Verma J, Monk BJ, Wolfson AH. New strategies for multimodality therapy in treating locally advanced cervix cancer. Semin Radiat Oncol 2016;26:344-8.

37. Schefter T, Winter K, Kwon JS, et al. RTOG 0417: efficacy of bevacizumab in combination with definitive radiation therapy and cisplatin chemotherapy in untreated patients with locally advanced cervical carcinoma. Int J Radiat Oncol Biol Phys 2014;88:101-5.

38. Gandhi AK, Sharma DN, Rath GK, et al. Early clinical outcomes and toxicity of intensity modulated versus conventional pelvic radiation therapy for locally advanced cervix carcinoma: a prospective randomized study. Int J Radiat Oncol Biol Phys 2013;87:542-8.
39. Chen CC, Lin JC, Jan JS, Ho SC, Wang L. Definitive intensitymodulated radiation therapy with concurrent chemotherapy for patients with locally advanced cervical cancer. Gynecol Oncol 2011;122:9-13.

40. Hasselle MD, Rose BS, Kochanski JD, et al. Clinical outcomes of intensity-modulated pelvic radiation therapy for carcinoma of the cervix. Int J Radiat Oncol Biol Phys 2011;80:1436-45.

41. Naik A, Gurjar OP, Gupta KL, Singh K, Nag P, Bhandari V. Comparison of dosimetric parameters and acute toxicity of intensity-modulated and three-dimensional radiotherapy in patients with cervix carcinoma: a randomized prospective study. Cancer Radiother 2016;20:370-6.

42. Mell LK, Sirak I, Wei L, et al. Bone marrow-sparing intensity modulated radiation therapy with concurrent cisplatin for stage IB-IVA cervical cancer: an International Multicenter Phase II Clinical Trial (INTERTECC-2). Int J Radiat Oncol Biol Phys 2017;97:536-45.

43. Noticewala SS, Li N, Williamson CW, et al. Longitudinal changes in active bone marrow for cervical cancer patients treated with concurrent chemoradiation therapy. Int J Radiat Oncol Biol Phys 2017:97:797-805.

44. Jadon $\mathrm{R}$, Pembroke $\mathrm{CA}$, Hanna $\mathrm{CL}$, et al. A systematic review of organ motion and image-guided strategies in external beam radiotherapy for cervical cancer. Clin Oncol (R Coll Radiol) 2014;26:185-96.

45. Lim K, Small W Jr, Portelance $L$, et al. Consensus guidelines for delineation of clinical target volume for intensity-modulated pelvic radiotherapy for the definitive treatment of cervix cancer. Int J Radiat Oncol Biol Phys 2011;79:348-55.

46. Castelnau-Marchand P, Chargari C, Maroun $P$, et al. Clinical outcomes of definitive chemoradiation followed by intracavitary pulsed-dose rate image-guided adaptive brachytherapy in locally advanced cervical cancer. Gynecol Oncol 2015;139:288-94.

47. Potter R, Georg P, Dimopoulos JC, et al. Clinical outcome of protocol based image (MRI) guided adaptive brachytherapy combined with 3D conformal radiotherapy with or without chemotherapy in patients with locally advanced cervical cancer. Radiother Oncol 2011;100:116-23.

48. Kang HC, Shin KH, Park SY, Kim JY. 3D CT-based high-doserate brachytherapy for cervical cancer: clinical impact on late rectal bleeding and local control. Radiother Oncol 2010;97:507-13.

49. Tan LT, Coles CE, Hart C, Tait E. Clinical impact of computed tomography-based image-guided brachytherapy for cervix cancer using the tandem-ring applicator - the Addenbrooke's experience. Clin Oncol (R Coll Radiol) 2009;21:175-82. 
50. Charra-Brunaud C, Harter $V$, Delannes $M$, et al. Impact of 3D image-based PDR brachytherapy on outcome of patients treated for cervix carcinoma in France: results of the French STIC prospective study. Radiother Oncol 2012;103:305-13.

51. Simpson DR, Scanderbeg DJ, Carmona R, et al. Clinical outcomes of computed tomography-based volumetric brachytherapy planning for cervical cancer. Int J Radiat Oncol Biol Phys 2015;93:150-7.

52. Han K, Milosevic M, Fyles A, Pintilie M, Viswanathan AN. Trends in the utilization of brachytherapy in cervical cancer in the United States. Int J Radiat Oncol Biol Phys 2013;87:111-9.

53. Gill BS, Lin JF, Krivak TC, et al. National Cancer Data Base analysis of radiation therapy consolidation modality for cervical cancer: the impact of new technological advancements. Int J Radiat Oncol Biol Phys 2014;90:1083-90.

54. Grover S, Harkenrider MM, Cho LP, et al. Image guided cervical brachytherapy: 2014 Survey of the American Brachytherapy Society. Int J Radiat Oncol Biol Phys 2016;94:598-604.

55. Potter R, Haie-Meder C, Van Limbergen E, et al. Recommendations from gynaecological (GYN) GEC ESTRO working group (II): concepts and terms in 3D image-based treatment planning in cervix cancer brachytherapy-3D dose volume parameters and aspects of 3D image-based anatomy, radiation physics, radiobiology. Radiother Oncol 2006;78:6777.

56. Potter R, Dimopoulos J, Georg P, et al. Clinical impact of $M R I$ assisted dose volume adaptation and dose escalation in brachytherapy of locally advanced cervix cancer. Radiother Oncol 2007;83:148-55.

57. Mayadev J, Viswanathan A, Liu Y, et al. American Brachytherapy Task Group Report: a pooled analysis of clinical outcomes for high-dose-rate brachytherapy for cervical cancer. Brachytherapy 2017;16:22-43.

58. Sturdza A, Potter R, Fokdal LU, et al. Image guided brachytherapy in locally advanced cervical cancer: Improved pelvic control and survival in RetroEMBRACE, a multicenter cohort study. Radiother Oncol 2016;120:428-33.

59. Tanderup K, Fokdal LU, Sturdza A, et al. Effect of tumor dose, volume and overall treatment time on local control after radiochemotherapy including MRI guided brachytherapy of locally advanced cervical cancer. Radiother Oncol 2016;120:441-6.

60. Kim H, Houser CJ, Kalash R, et al. Workflow and efficiency in MRI-based high-dose-rate brachytherapy for cervical cancer in a high-volume brachytherapy center. Brachytherapy 2018;17:753-60.

61. Mendez LC, Leung $E$, Cheung $P$, Barbera L. The role of stereotactic ablative body radiotherapy in gynaecological cancers: a systematic review. Clin Oncol (R Coll Radiol) 2017;29:378-84.

62. Marnitz S, Kohler C, Budach V, et al. Brachytherapy-emulating robotic radiosurgery in patients with cervical carcinoma. Radiat Oncol 2013;8:109.

63. Hsieh $\mathrm{CH}$, Tien $\mathrm{HJ}$, Hsiao SM, et al. Stereotactic body radiation therapy via helical tomotherapy to replace brachytherapy for brachytherapy-unsuitable cervical cancer patients: a preliminary result. Onco Targets Ther 2013;6:59-66.

64. Haas JA, Witten MR, Clancey O, Episcopia K, Accordino D, Chalas E. CyberKnife boost for patients with cervical cancer unable to undergo brachytherapy. Front Oncol 2012;2:25.

65. Kubicek GJ, Xue J, Xu O, et al. Stereotactic body radiotherapy as an alternative to brachytherapy in gynecologic cancer. Biomed Res Int 2013;2013:898953.

66. Molla M, Escude L, Nouet $P$, et al. Fractionated stereotactic radiotherapy boost for gynecologic tumors: an alternative to brachytherapy? Int J Radiat Oncol Biol Phys 2005;62:118-24.

67. Wulf J, Hadinger U, Oppitz U, Thiele W, Flentje M. Stereotactic boost irradiation for targets in the abdomen and pelvis. Radiother Oncol 2004;70:31-6.

68. Mazzola R, Ricchetti F, Fiorentino A, et al. Weekly cisplatin and volumetric-modulated arc therapy with simultaneous integrated boost for radical treatment of advanced cervical cancer in elderly patients: feasibility and clinical preliminary results. Technol Cancer Res Treat 2017;16:310-5.

69. Kim H, Kim JY, Kim J, et al. Current status of brachytherapy in Korea: a national survey of radiation oncologists. J Gynecol Oncol 2016;27:e33.

70. Viswanathan AN, Thomadsen B; American Brachytherapy Society Cervical Cancer Recommendations Committee; American Brachytherapy Society. American Brachytherapy Society consensus guidelines for locally advanced carcinoma of the cervix. Part I: general principles. Brachytherapy 2012;11:33-46

71. Cibula D, Potter R, Planchamp F, et al. The European Society of Gynaecological Oncology/European Society for Radiotherapy and Oncology/European Society of Pathology Guidelines for the management of patients with cervical cancer. Int J Gynecol Cancer 2018;28:641-55.

72. Gouy S, Morice P, Narducci F, et al. Prospective multicenter study evaluating the survival of patients with locally advanced cervical cancer undergoing laparoscopic paraaortic lymphadenectomy before chemoradiotherapy in the era of positron emission tomography imaging. J Clin Oncol 2013;31:3026-33. 
73. Dabi $Y$, Simon $V$, Carcopino $X_{1}$ et al. Therapeutic value of surgical paraaortic staging in locally advanced cervical cancer: a multicenter cohort analysis from the FRANCOGYN study group. J Transl Med 2018;16:326.

74. Moore KN, Java JJ, Slaughter $\mathrm{KN}$, et al. Is age a prognostic biomarker for survival among women with locally advanced cervical cancer treated with chemoradiation? An NRG Oncology/Gynecologic Oncology Group ancillary data analysis. Gynecol Oncol 2016;143:294-301.

75. Sharma C, Deutsch I, Horowitz DP, et al. Patterns of care and treatment outcomes for elderly women with cervical cancer. Cancer 2012;118:3618-26. 